\title{
X-Ray Evidence for Wind Instabilities
}

\author{
Joseph J. MacFarlane, Joseph P. Cassinelli, and D.H. Cohen
}

University of Wisconsin - Madison

Hot stars are known to emit X-rays with $L_{X} / L_{b o l} \sim 10^{-7}$ for $\mathrm{O}$ stars, falling to $\sim 10^{-9}$ for B3 stars. These stars also lose mass at large rates through their high-speed winds. Over the years, several types of production mechanisms have been proposed to explain the X-ray emission from $\mathrm{O}$ stars, with source locations ranging from very near the stellar surface to very far from the star. A coronal X-ray source was originally proposed (Cassinelli and Olson 1979) to explain the presence of anomalously high ionization stages observed as P Cygni line profiles in the UV spectra of O stars. At the other extreme, Chlebowski (1989) suggested that the X-rays of O stars originate far from the star, and are produced by the interaction of the stellar wind with circumstellar matter. A model in which shocks forming due to instabilities in the line-driven winds of $O$ stars was proposed by Lucy (1982), and studied in detail by Owocki et al. (1988), Cooper (1994), and Feldmeier (1996). In this case, the $\mathrm{X}$-ray emission originates in a large number of shock-heated regions distributed throughout the wind. The shocked-wind model has also been shown to be consistent with the X-ray emission from early-B stars, such as $\tau$ Sco (MacFarlane and Cassinelli 1989). However, it appears difficult for shocked wind models to explain the X-ray emission from B3 and later stars because of their presumed low mass loss rates (Cohen et al. 1997).

Here, we discuss evidence that suggests that the X-rays we observe from hot stars originate from shock-heated plasma created by radiatively-driven wind instabilities. The observational evidence for this comes from observations at a variety of wavelengths, including: moderate-resolution X-ray spectra, EUV line emission, and UV P-Cygni profiles. The lack of significant bound-free absorption due to an overlying cool wind (Cassinelli et al. 1981; Corcoran et al. 1993) suggests that a significant fraction of the X-rays must be emitted from regions significantly above the base of $\mathrm{O}$ star winds. A detailed analysis of the dependence of the wind ionization distribution and resulting $O$ VI P Cygni profile on the X-ray source distribution for $\zeta$ Pup was carried out by MacFarlane et al. (1994). They found that the UV O VI profile was not consistent with: (1) models in which the X-ray source was located at a radius much above $\sim$ several stellar radii; and (2) coronal models, unless the mass loss rate for $\zeta$ Pup is a factor of $\sim 3$ to 5 lower than the value deduced from radio observations. More recently, EUVE observations of the B2 II star $\epsilon$ CMa (Cassinelli et al. 1995), along with a combined analysis of its ROSAT and EUVE data (Cohen et al. 1996), indicate the presence of a moderate amount of wind attenuation for this relatively low mass-loss rate star, as well as a multitemperature plasma X-ray source. Thus, at a variety 
of wavelengths, observations and analysis provide significant evidence for a shocked-wind $\mathrm{X}$-ray source for $\mathrm{O}$ and early-B stars.

\section{Discussion}

S. Owocki: In your model of $\zeta$ Pup, although you may need to have the Xrays begin close to the star, the $\mathrm{X}$-rays one sees come from quite far away, e.g., $\mathrm{r} \geq 10 R_{\star}$. This suggests that the radial fall-off of your assumed X-ray source should also be an important parameter. Thus, if you vary this, you might be able to match the observed X-rays with a source that has less effect on the inner ionisation.

A. Moffat: In your model, you assume homogeneity of the wind. However, we know that hot-star winds are highly clumped (e.g., Eversberg et al. 1997 on $\zeta$ Pup). This might have a strong effect on your models. In fact, the clumps may themselves be the source of the X-ray flux via shocks.

J. MacFarlane: At present, this is a shortfall in our modelling. It would indeed be good to do calculations to study the effect of clumping.

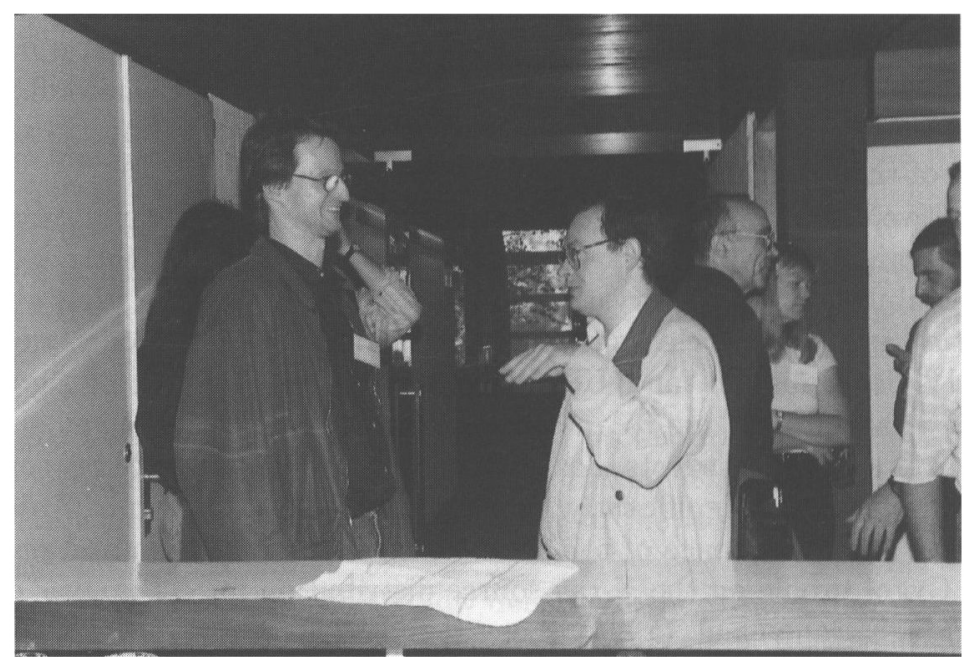

Thomas Berghöfer and Ronny Blomme 